\title{
Controle da dengue na estação seca da cidade de Curvelo-MG através de um modelo preditivo
}

\author{
Lillia S. Barsante, Flávia S. Cordeiro, \\ Rodrigo T. N. Cardoso, José L. Acebal \\ Pós-Graduação Modelagem Matemática e Computacional, PPGMMC, CEFET-MG, \\ 30510-000, Belo Horizonte, MG \\ E-mail: lilliabarsante@gmail.com, fscordeiro.mat@gmail.com, \\ rodrigoc@des.cefetmg.br, acebal@dppg.cefetmg.br \\ Kelly S. Paixão , Álvaro E. Eiras \\ UFMG - Departamento de Biologia, Laboratório de Ecologia Química-LabEQ \\ 31270-901, Belo Horizonte, MG \\ E-mail: kellypaixaoufmg@gmail.com, alvaro.eiras@gmail.com
}

\begin{abstract}
Resumo: Apesar do crescente orçamento destinado ao controle do vetor da dengue, segundo a Organização Mundial de Saúde, 50 a 100 milhões de pessoas anualmente contraem a doença. Buscando minimizar os custos dos gestores no controle da dengue, torna-se importante determinar a melhor época do ano para se aplicar ações de controle do vetor. Desta forma, através de um modelo matemático que descreve a dinâmica populacional do vetor da dengue sob a influência de pluviosidade, foi verificado para a cidade de Curvelo (Minas Gerais, Brasil) a vantagem de se realizar ações de controle do vetor da dengue durante a estação seca, em vez do procedimento comum adotado pelos programas de controle de vetores, em que as ações de controle são aplicados principalmente na época das chuvas.
\end{abstract}

Palavras-chave: Modelo Matemático, Pluviosidade, Controle

\section{Introdução}

De acordo com dados da Organização Mundial de Saúde - OMS, a dengue atinge entre 50 e 100 milhões de pessoas anualmente e mais de 2.5 bilhões estão vivendo em áreas onde existe o risco de se contrair a doença. A cadeia epidemiológica da dengue é complexa e depende da interação do vetor, do vírus e do humano e está associada a aspectos ecológicos e fatores climáticos. No Brasil, em 2013, foram registrados cerca de 1.477.000 casos da doença. Como ainda não existe uma vacina que possa atuar efetivamente contra os sorotipos conhecidos e o tratamento da doença é apenas sintomático, o controle da dengue se restringe no controle do vetor [7].

O Aedes aegypti é o principal vetor da dengue. Este vetor, nativo do Velho Mundo, é cosmopolita e se encontra em regiões tropicais e subtropicais. Esse mosquito chegou nas Américas, provavelmente, durante o tráfego de escravos entre os séculos XVI e XIX e hoje, devido à sua estreita associação com a população humana, já se encontra distribuído pelos vinte e sete estados brasileiros [2].

As fêmeas desse vetor necessitam se alimentar de sangue de vertebrados para a obtenção de proteínas fundamentais para que ocorra a maturação dos ovócitos em seus ovários e, consequentemente, a postura de ovos viáveis. O A. aegypti possui hábito diurno com atividade hematofágica apresentando picos ao amanhecer e no crepúsculo vespertino. O fato de a fêmea necessitar realizar mais de um repasto sanguíneo para a postura de ovos viáveis tem grande im- 
portância epidemiológica, pois favorece a infecção do vetor e também a transmissão de patógenos para os hospedeiros vertebrados suceptíveis [2].

A influência de variáveis metereológicas na dinâmica das populações do ciclo de vida do $A$. aegypti e na transmissão da dengue têm sido abordados em diversos trabalhos como ([5]; [6]; [1]; $[3])$.

Neste trabalho, um modelo matemático preditivo para dinâmica das populações do ciclo de vida do A. aegypti sob influência da pluviosidade foi usado para avaliar o efeito de se realizar ações de controle do A. aegypti durante a estação seca da cidade de Curvelo (Minas Gerais, Brasil).

\section{Modelo}

O modelo baseado em um sistema de equações diferenciais ordinárias não-lineares para descrever a dinâmica das populações do ciclo de vida do $A$. aegypti diante da variação da pluviosidade foi descrito em [1]. As variáveis deste modelo são $X(t)=\left(E(t) ; A(t) ; F_{1}(t) ; F_{2}(t)\right)$, sendo que $E(t)$ representa a população de ovos, $A(t)$ representa a população aquática (larvas + pupas), $F_{1}(t)$ representa a população de fêmeas pré-repasto sanguíneo, $F_{2}(t)$ representa a população de fêmeas pós-repasto sanguíneo, de modo que a dinâmica destas populações é dada por:

$$
\left\{\begin{aligned}
\frac{d E}{d t} & =\phi(p)\left(1-\frac{E(t)}{C(t)}\right) F_{2}(t)-\left(\alpha_{1}(p)+\mu_{E}(p)+c_{E}(t)\right) E(t) \\
\frac{d A}{d t} & =\alpha_{1}(p) E(t)-\left(\alpha_{2}(p)+\mu_{A}(p)+c_{A}(t)\right) A(t) \\
\frac{d F_{1}}{d t} & =\alpha_{2}(p) A(t)-\left(\alpha_{3}(p)+\mu_{F_{1}}(p)+c_{F_{1}}(t)\right) F_{1}(t), \\
\frac{d F_{2}}{d t} & =\alpha_{3}(p) F_{1}(t)-\left(\mu_{F_{2}}(p)+c_{F_{2}}(t)\right) F_{2}(t), \\
\phi, & \alpha_{1}, \alpha_{2}, \alpha_{3}, \mu_{E}, \mu_{A}, \mu_{F_{1}}, \mu_{F_{2}}, C, c_{E}, c_{A}, c_{F_{1}}, c_{F_{2}} \geq 0, \quad \forall p, t \in \mathbb{R}_{+} .
\end{aligned}\right.
$$

O parâmetro $\phi$ é a taxa de oviposição intrínseca por unidade de fêmeas pós-repasto sanguíneo; $C$ é a capacidade do meio em termos de disponibilidade de nutrientes, de espaço e outros; $\alpha_{1}$ é taxa por unidade de ovos que passa para a população aquática; $\alpha_{2}$ é a taxa por unidade da população aquática que transforma em fêmeas pré-repasto sanguíneo; $\alpha_{3}$ é a taxa por unidade de fêmeas pré-repasto sanguíneo que transformam em fêmeas pós-repasto sanguíneo; $\mu$ e $c$ são as taxas por unidade de indivíduo que morre naturalmente e adicionalmente devido a ação de controle da respectiva população do ciclo de vida do $A$. aegypti. Os parâmetros $t$ e $p$ representam, respectivamente, tempo e pluviosidade.

Descrevemos a dependência paramétrica dos coeficientes do modelo (1) com a pluviosidade através da lei de potência (2), dada por:

$$
\boldsymbol{\pi}=\boldsymbol{\pi}_{\min }+\frac{\left(\boldsymbol{\pi}_{\max }-\boldsymbol{\pi}_{\min }\right)}{\left(p_{\max }-p_{\min }\right)^{r}}\left(p-p_{\min }\right)^{r},
$$

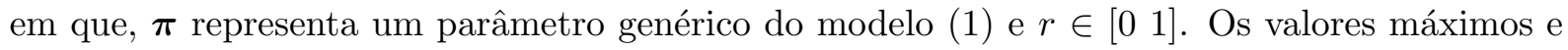
mínimos dos parâmetros do modelo (1) estão associados ao valor máximo e mínimo da pluviosidade de uma determinada região.

Se os parâmetros do modelo (1) não dependem do tempo, pode-se identificar um ponto de equilíbrio trivial $P_{0}$,

$$
\left(E^{*}, A^{*}, F_{1}^{*}, F_{2}^{*}\right)=(0,0,0,0),
$$


e um ponto de equilíbrio não trivial $P_{1}$,

$$
\left(E^{* *}, A^{* *}, F_{1}^{* *}, F_{2}^{* *}\right)=\left\{\begin{aligned}
E^{* *} & =C\left(1-\frac{1}{R_{0}}\right), \\
A^{* *} & =\frac{\alpha_{1}}{\left(\alpha_{2}+\mu_{A}+c_{A}\right)} E^{* *}, \\
F_{1}^{* *} & =\frac{\alpha_{2}}{\left(\alpha_{3}+\mu_{F_{1}}+c_{F_{1}}\right)} A^{* *}, \\
F_{2}^{* *} & =\frac{\alpha_{3}}{\left(\mu_{F_{2}}+c_{F_{2}}\right)} F_{1}^{* *},
\end{aligned}\right.
$$

em que, $R_{0}$ é análogo ao número de reprodução básica:

$$
R_{0}=\frac{\phi}{\left(\alpha_{1}+\mu_{E}+c_{E}\right)} \frac{\alpha_{1}}{\left(\alpha_{2}+\mu_{A}+c_{A}\right)} \frac{\alpha_{2}}{\left(\alpha_{3}+\mu_{F_{1}}+c_{F_{1}}\right)} \frac{\alpha_{3}}{\left(\mu_{F_{2}}+c_{F_{2}}\right)} .
$$

Pode-se observar que a equação (5) é sempre maior que zero, pois todos os parâmetros são positivos. Se $R_{0}=1$ implica que $P_{0}=P_{1}$, onde temos um de ponto de bifurcação transcrítica [3] e se $R_{0}>1$ o ponto (4) é positivo.

O estudo da estabilidade destes pontos críticos foi realizado através dos critérios de RouthHurwitz. O resultado encontrado foi que se $0<R_{0}<1, P_{0}$ é localmente e assintoticamente estável e $P_{1}$ é instável e se $R_{0}>1, P_{0}$ é estável e $P_{1}$ é localmente e assintoticamente estável ([1]; $[3])$.

\section{Resultados Obtidos}

Experimentos computacionais foram realizados com o modelo (1) utilizando dados amostrais de pluviosidade acumulada semanalmente, referentes às semanas epidemiológicas 8 a 51 do ano de 2009 para cidade de Curvelo (Minas Gerais, Brasil). Estes dados foram obtidos do Instituto Nacional de Pesquisas Espaciais (INPE, Brasil) via LabEQ.

Os valores máximos e mínimos dos coeficientes entomológicos $\left(\phi, \alpha_{1}, \alpha_{2}, \alpha_{3}, \mu_{F_{1}}\right.$ e $\left.\mu_{F_{2}}\right)$ são mostrados na Tabela 1. Adotamos o valor constante de 0.01 e 0.164 , respectivamente, para os coeficientes entomológicos $\mu_{E}$ e $\mu_{A}$. A unidade dos coeficientes entomológicos é dias ${ }^{-1}$. Alguns desses valores foram obtidos a partir de [5] e [6], e outros foram estimados por especialistas da área biológica.

\begin{tabular}{cccccc}
\hline Parâmetro & Faixa & Parâmetro & Faixa & Parâmetro & Faixa \\
\hline$\phi$ & $0.56-11.2$ & $\alpha_{1}$ & $0.01-0.5$ & $\alpha_{2}$ & $0.06-0.16$ \\
$\alpha_{3}$ & $0.333-1.0$ & $\mu_{F_{1}}$ & $0.043-0.17$ & $\mu_{F_{2}}$ & $0.057-0.17$ \\
\hline
\end{tabular}

Tabela 1: Faixa de parâmetros adotados no modelo (1).

A capacidade do meio $C$ foi fixada em 1, fazendo com que o tamanho das populações do modelo (1) variassem como frações da unidade.

O modelo (1) foi implementado usando o método de Runge - Kutta de quarta ordem em MATLAB R2009b(MathWorks Inc., mathwork.com, Natick, MA, EUA). Nesta implementação, (i) a condição $R_{0}>1$ foi verificada para cada um dos valores $p$; (ii) as ações de controle foram definidas para serem constante $\left(c_{E}=c_{A}=c_{F_{1}}=c_{F_{2}}=0.7\right)$ ao longo de apenas uma semana epidemiológica; e (iii) adotamos o valor de $P_{1}$ correspondente a primeira semana epidemiológica de estudo como o valor de condição inicial.

As Figuras 1 a 4 ilustram a dinâmica das populações do ciclo de vida do A. aegypti com a pluviosidade acumulada semanalmente para a cidade de Curvelo (Minas Gerais, Brasil), sem aplicação de ações de controle durante o período de estudo. Os dados foram normalizados para proporcionar uma métrica de comparação. 


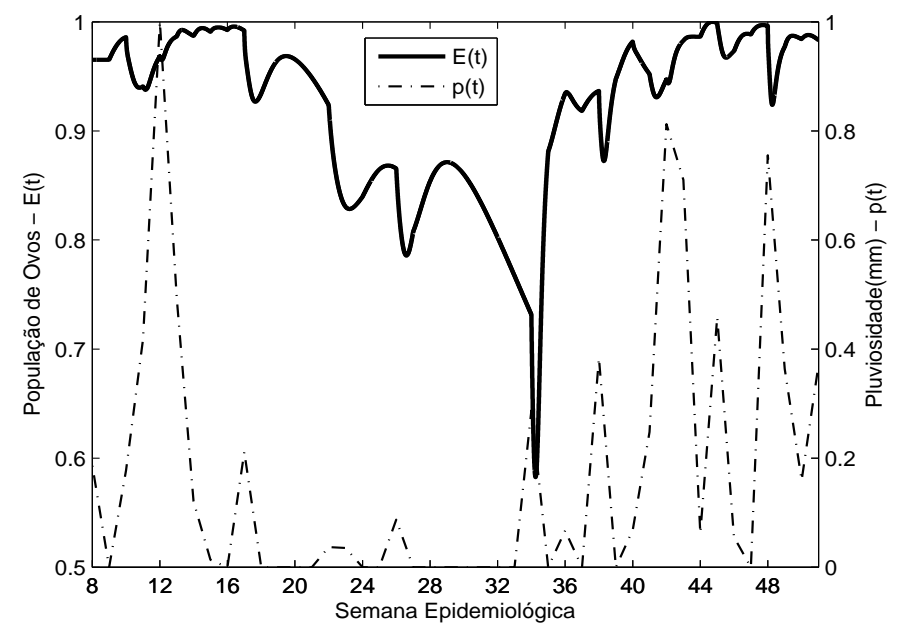

Figura 1: Evolução da população $E(t) \operatorname{com} p(t)$, sem aplicação de controle durante o período de estudo.

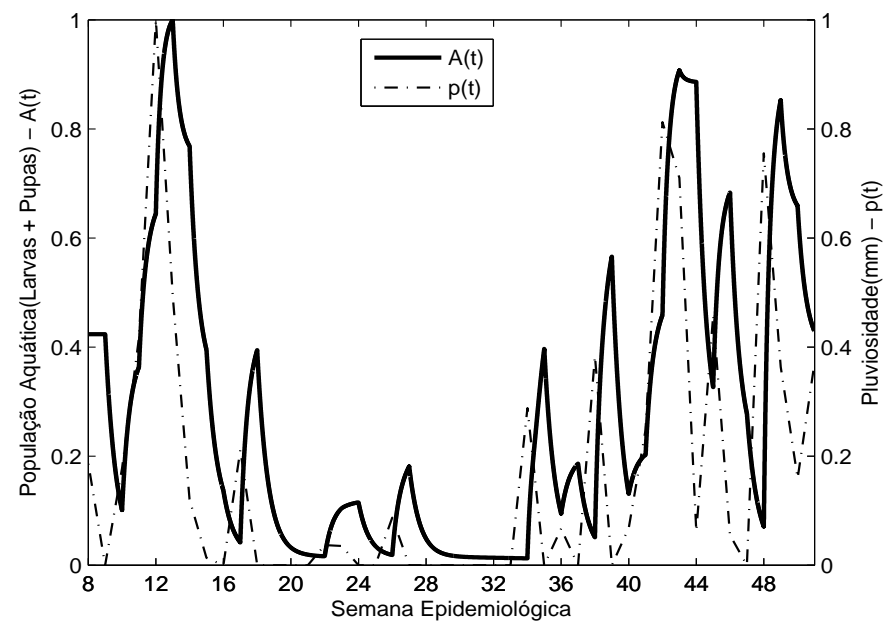

Figura 2: Evolução da população $A(t) \operatorname{com} p(t)$, sem aplicação de controle durante o período de estudo.

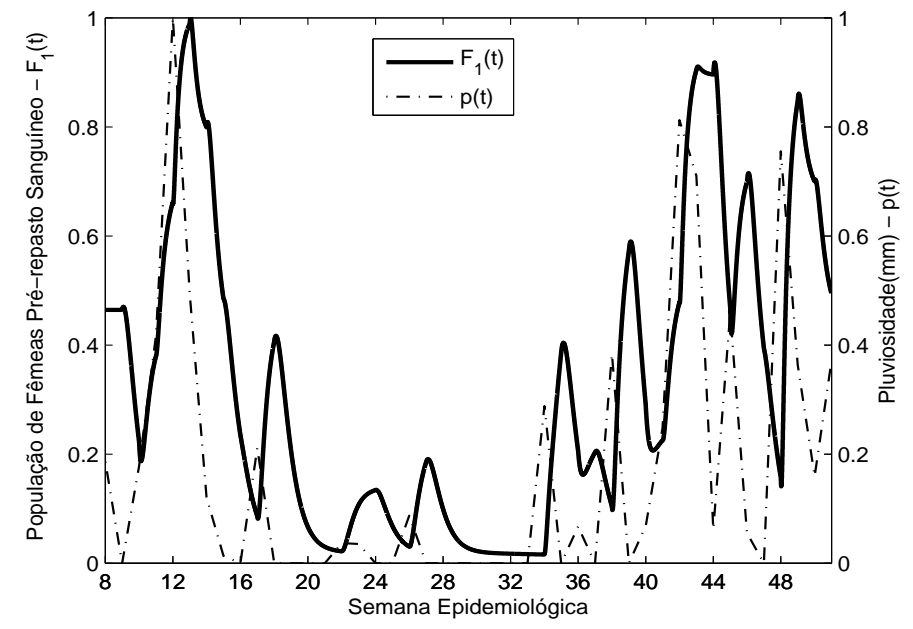

Figura 3: Evolução da população $F_{1}(\mathrm{t}) \operatorname{com} p(t)$, sem aplicação de controle durante o período de estudo. 


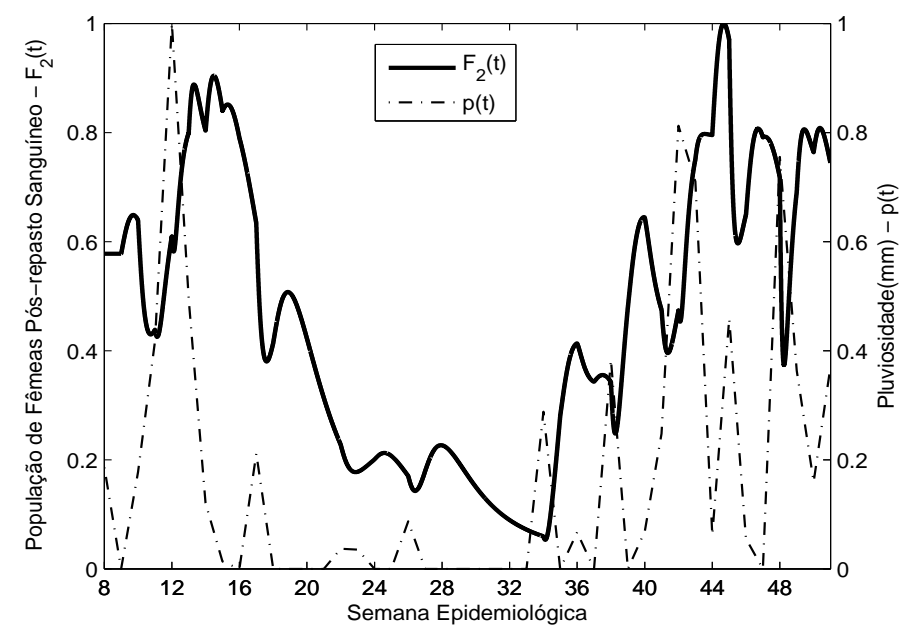

Figura 4: Evolução da população $F_{2}(\mathrm{t}) \operatorname{com} p(t)$, sem aplicação de controle durante o período de estudo.

Percebemos na Figura 1 que um pequeno período chuvoso é o suficiente para manter a população $E(t)$ elevada, em média, nas próximas duas semanas. Nos longos períodos de seca, como ocorrido entre as semanas 27 e 33, esta população sofre um decaimento significativo. Nas Figuras 2 e 3 ocorre um grande coincidência de picos entre as populações $A(t)$ e $F_{1}(t)$ com os índices pluviométricos, evidenciando a influência da pluviosidade na dinâmica das populações do ciclo de vida do $A$. aegypti. Finalmente, observamos através da Figura 4 que a população $F_{2}(t)$ também acompanha os índices pluviométricos, no entanto, o período de seca, causa um decaimento mais suave na população.

A validação do modelo ocorreu através da comparação qualitativa entre os resultados dos experimentos computacionais da população $F_{2}(t)$ do modelo $(1)$ e dados amostrais de Índice Médio de Fêmeas Aedes - IMFA. Este índice corresponde a razão entre o número de fêmeas $A$. aegypti em fase de oviposição capturadas pela armadilha MosquiTRAP ${ }^{\circledR}$ e o número total de armadilhas instaladas na região de estudo [4]. Os dados amostrais de $I M F A$, referente ao período de estudo, foram obtidos da Ecovec ${ }^{\circledR}$ via LabEQ. Desta forma, definimos uma nova população, $f_{2}(t)$, obtida a partir da população $F_{2}(t)$, por meio de uma função de correlação cruzada. No eixo x foi feita uma translação de $F_{2}(t)$, com o objetivo de coincidir o maior número de picos entre $F_{2}(t)$ e IMFA. No eixo y foi feito um ajuste pelo método dos mínimos quadrados com o intuito de comparar a área sob as curvas, onde foi considerado $f_{2}=a F_{2}, a \in \mathbb{R}$ e $D=\left(I M F A-f_{2}\right)^{2}$. O melhor ajuste foi obtido com $r=0.85$ para $\left(\phi, \alpha_{1}, \alpha_{2}, \alpha_{3}\right), r=1$ para $\left(\mu_{F_{1}}, \mu_{F_{2}}\right)$ e $r=0$ para $\left(\mu_{A}, \mu_{E}\right)$.

Observe na Figura 5 que existem coincidências na posição dos picos e vales de $f_{2}(t)$ e o $I M F A$. Além disso, há regiões onde $f_{2}(t)$ não consegue atingir o valor experimental de captura, ao passo que em outras regiões $f_{2}(t)$ excede os dados experimentais. Esta comparação pode ser considerada satisfatória, uma vez que desconsideramos a influência da temperatura e da umidade na dinâmica das populações do ciclo de vida do A. aegypti descritas no modelo (1) e os dados amostrais de captura podem sofrer influência de diversas formas, como metereológicas e humanas.

De acordo com especialistas da área biológica, o controle do A. aegypti deve ser intensificado nas estações frias e secas, em vez do procedimento comum adotado pelos programas de controle de vetores, em que as ações de controle são aplicados principalmente na época das chuvas, a fim de minimizar o tamanho da população do $A$. aegypti e reduzir o número de infecções anuais de 


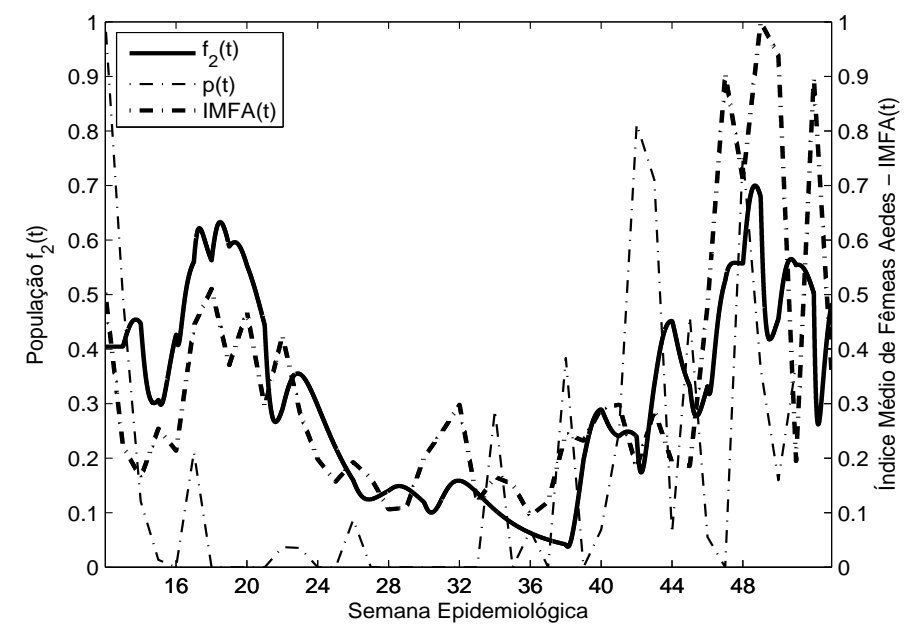

Figura 5: Comparação entre $f_{2}(t), I M F A$ e $p(t)$, sem aplicação de controle durante o período de estudo.

dengue no verão. Desta forma, focamos a análise do efeito desta ação de controle, apenas na população de fêmeas pós-repasto sanguíneo, pois elas são responsável pela transmissão do virús da dengue e são semelhantes as fêmeas capturadas pela armadilha MosquiTRAP ${ }^{\circledR}$.

Primeiramente, avaliamos a população $F_{2}(t)$ realizando controle em uma semana de baixa pluviosidade contra o caso de não realizar controle em nenhuma semana epidemiológica $\left(F_{2}(t)_{S B P} \times\right.$ $\left.F_{2}(t)_{S S C}\right)$. Em segundo lugar, avaliamos a população $F_{2}(t)$ realizando controle em uma semana de alta pluviosidade contra o caso de não realizar controle em nenhuma semana epidemiológica $\left(F_{2}(t)_{S A P} \times F_{2}(t)_{S S C}\right)$. Para avaliar a altura dos picos de depreciação e o tempo de latência destes controles na população $F_{2}(t)$ definimos a seguinte métrica:

$$
\Delta=\frac{\left|\int_{I} \frac{\left[F_{2}(t)_{S B P}\right]-\left[F_{2}(t)_{S S C}\right]}{\left[F_{2}(t)_{S S C}\right]} d t\right|-\left|\int_{I} \frac{\left[F_{2}(t)_{S A P}\right]-\left[F_{2}(t)_{S S C}\right]}{\left[F_{2}(t)_{S S C}\right]} d t\right|}{\left|\int_{I} \frac{\left[F_{2}(t)_{S A P}\right]-\left[F_{2}(t)_{S S C}\right]}{\left[F_{2}(t)_{S S C}\right]} d t\right|} \times 100 \% .
$$

A Tabela 2 ilustra a diferença relativa percentual $\Delta$ da área da curva da população $F_{2}(t)$ realizando controle em uma semana de baixa pluviosidade contra o controle realizado em uma semana de alta pluviosidade para a cidade de Curvelo (Minas Gerais, Brasil).

\begin{tabular}{cccccc}
\hline (SBP-SAP $)$ & $\Delta$ & $($ SBP-SAP $)$ & $\Delta$ & $($ SBP-SAP $)$ & $\Delta$ \\
\hline $19-12$ & $960.41 \%$ & $30-12$ & $560.43 \%$ & $37-12$ & $215.15 \%$ \\
$19-43$ & $933.23 \%$ & $30-43$ & $543.49 \%$ & $37-43$ & $207.07 \%$ \\
$19-48$ & $647.03 \%$ & $30-48$ & $365.25 \%$ & $37-48$ & $122.01 \%$ \\
\hline
\end{tabular}

Tabela 2: Diferença relativa percentual da área da população $F_{2}(t)$ : controle SBP $\times$ controle SAP.

Analisando a tabela 2, observamos que ações de controle realizadas em semanas epidemiológicas de baixa pluviosidade é mais eficaz na redução da população $F_{2}(t)$ do que as realizadas em semanas epidemiológicas de alta pluviosidade. Observe que o controle realizado na semana epidemiológica $19(\mathrm{SBP})$ foi $933.23 \%$ vezes maior que o realizado na semana $43(\mathrm{SAP})$.

\section{Conclusão}

Os resultados mostram que o modelo (1) permite conduzir ações eficientes de controle do $A$. aegypti quando realizado nas semanas secas da cidade de Curvelo (Minas Gerais, Brasil). Este 
fato é extremamente relevante, pois iremos reduzir a população de ovos que provavelmente iriam eclodir na próxima estação chuvosa.

Trabalhos em andamento já incluem juntamente com a pluviosidade, o efeito da temperatura nos parâmetros entomológicos do modelo (1). Trabalhos futuros irão avaliar métodos de otimização para: (i) proporcionar o refinamento da dependência dos parâmetros entomológicas do modelo com os parâmetros metereológicos; e (ii) definir os intervalos de tempo em que as ações de controle devem ser realizadas pelos gestores públicos. Iremos também incluir o efeito da umidade, juntamente com a pluviosidade e temperatura, nos parâmetros entomológicos do modelo (1).

\section{Referências}

[1] L.S. Barsante, "Dependência entre Pluviosidade e População de Fêmeas Aedes aegypti Grávidas Descritas Através de um Sistema Dinâmico não Linear", Dissertação de Mestrado, PPGMMC -CEFET-MG, 2012.

[2] R.A.G.B., Consoli e R.L., Oliveira, "Principais Mosquitos de Importância Sanitária no Brasil", FIOCRUZ, Rio de Janeiro, 1994.

[3] F.S. Cordeiro, "Modelo Preditivo para a População de Mosquitos Aedes aegypti sob Influência de Variáveis Climáticas: Temperatura e Pluviosidade", Dissertação de Mestrado, PPGMMC -CEFET-MG, 2014.

[4] Á.E. Eiras and M.C. Resende, Preliminary evaluation of the "Dengue-MI" technology for Aedes aegypti monitoring and control, Cad. Saúde Pública 25(1) (2009) S45-S58.

[5] C.P. Ferreira e H.M. Yang, Estudo da Transmissão da Dengue entre os Indivíduos em Interação com a População de Mosquitos Aedes aegypti, TEMA Tend. Mat. Apl. Comput $4(3)$ (2003) 323-332.

[6] S.T.R. Pinho, C.P. Ferreira, L. Esteva, F.R. Barreto, V.C. Morato e Silva and M.G.L. Teixeira, Modelling the dynamics of dengue real epidemics, Philosophical Transactions of the Royal Society A: Mathematical, Physical and Engineering Sciences, 368(1933) (2010) 5679-5693.

[7] WORLD HEALTH ORGANIZATION (WHO) 2013. Dengue and Severe Dengue. Disponível em http://www.who.int/mediacentre/factsheets/fs117/en/ acesso em 26/02/2014. 